\title{
Dr. Cord Eberspächer
}

Cord Eberspächer ist seit Januar 2010 Direktor des Konfuzius-Instituts an der Heinrich-Heine-Universität Düsseldorf. Der Historiker und Sinologe hat in Oldenburg, Hamburg, Leiden und Peking studiert und promovierte 2003 mit einer Arbeit über deutsche Kanonenbootpolitik in China. Er hat von 1995 bis 2004 als Historiker und Pressereferent bei der Oldenburgischen Landschaft gearbeitet und war anschließend von 2005 bis 2008 am Geheimen Staatsarchiv Preußischer Kulturbesitz in Berlin mit dem Projekt „Preußen-Deutschland und China 1842-1911“ befasst. Seine Forschungen zur chinesischen Geschichte des 19. und frühen 20. Jahrhunderts baute er dann durch seine Tätigkeit an der University of Bristol im Rahmen des Projekts „Colonialism in Comparative Perspective. Tianjin under Nine Flags" weiter aus. Er ist Mitinitiator des europäisch-chinesischen Projekts "China-related Documents in European Archives". 


\section{CORD EBERSPÄCHER}

\section{Kennen Sie Konfuzius? \\ Über 300 Konfuzius-Institute verbreiten chinesische Kultur und Sprache weltweit - das Düsseldorfer Institut gehörte zu den ersten}

Vermittlung von Sprache und Kultur des eigenen Landes ist seit Langem Bestandteil der Kulturpolitik vieler westlicher Länder. Ob British Council, Instituto Cervantes oder Goethe-Institut, alle sind weltweit bekannte Marken, deren Geschichte teilweise schon über 70 Jahre zurückreicht. Aber der Westen steht damit nicht mehr allein. China hat seit 2004 seine eigenen Kulturinstitute ins Leben gerufen und analog zu seinen Vorbildern nach einer chinesischen Geistesgröße benannt: Konfuzius-Institut.

Düsseldorf hat seit 2006 ein solches chinesisches Kulturinstitut und die beiden ersten Direktoren, Dr. Peter Hachenberg und Prof. Dr. Li Xuetao, haben dies damals zum Anlass genommen, in einem Beitrag für das Jahrbuch der Heinrich-Heine-Universität Gründung, Programm und Perspektiven für diese neue Einrichtung zu umreißen. ${ }^{1}$ Inzwischen kann das Konfuzius-Institut bald seinen vierten Geburtstag feiern und es haben sich zahlreiche Veränderungen ergeben; Anlass genug, auf die noch junge Geschichte der Konfuzius-Institute und insbesondere des Standortes in Düsseldorf zurückzublicken und Perspektiven und Pläne für die Zukunft zu erörtern.

\section{Konfuzius-Institute: Eine junge Entwicklung}

Alle Konfuzius-Institute sind noch sehr jung, das erste wurde 2004 in Seoul gegründet. Doch auch hier nimmt China eine rasante Entwicklung: Inzwischen gibt es weltweit über 300 Konfuzius-Institute, auf sämtlichen Kontinenten. Möglich wurde diese atemberaubend schnelle Entwicklung durch ein bislang einmaliges Modell: KonfuziusInstitute sind gemeinsame Unternehmen gleichberechtigter Partner - Joint Ventures zwischen China und dem jeweiligen Gastland. Unter dem Dach von Hanban, dem Konfuzius-Hauptquartier, werden die Institute von zwei Partnern getragen, einer Universität in China und einer im Ausland. Das ist ein bemerkenswerter Schritt von chinesischer Seite, denn damit haben die ausländischen Partner natürlich auch ein Mitspracherecht bei der Gestaltung des Programms - Konfuzius-Institute sind also keineswegs ein simples Sprachrohr des offiziellen China.

Diese Konstruktion war aber sicher ein entscheidender Grund dafür, dass sich die Konfuzius-Institute in solcher Geschwindigkeit verbreitet haben. Obwohl China bereit ist, erhebliche Summen in dieses kulturelle Großprojekt zu investieren, ist die Einrichtung eines einzelnen Instituts vergleichsweise preiswert: als „Grundbausatz" genügen ein chinesischer Direktor, ein Sprachlehrer, einige Pakete Lehrbücher und ein Zuschuss

\footnotetext{
1 Vgl. Hachenberg und Li (2007).
} 
für das Kulturprogramm. Räumlichkeiten und lokales Personal kommen dann vom ausländischen Partner. Auch wenn dieser „Grundbausatz" natürlich nur ein Anfang sein kann, wird schnell deutlich, dass der finanzielle Aufwand deutlich geringer ist als für ein vergleichbares voll ausgestattetes Goethe-Institut.

Konfuzius-Institute können sehr unterschiedlich sein. Zunächst liegt das an den örtlichen Bedingungen, wie der Größe des Landes, der Anzahl der Chinesen vor Ort, den wirtschaftlichen Bedingungen und nicht zuletzt der politischen Situation. So sind die politischen Rahmenbedingungen dafür entscheidend, dass das Konfuzius-Institut in Kabul bewaffnete Wachmänner vor der Tür stehen hat - genauso wie es in Afghanistan praktisch unmöglich ist, lokale Lehrkräfte mit ausreichenden Chinesischkenntnissen zu finden. Dagegen hat beispielsweise das Konfuzius-Institut in Bratislava zwar auch keine glänzenden Ausgangsbedingungen vor Ort, spielt aber eine herausragende Rolle für die Entwicklung der slowakischen Beziehungen zu China und bekommt somit entsprechende Aufmerksamkeit und Unterstützung. In einigen Bundesstaaten der USA bestehen bereits flächendeckend Konfuzius-Klassenräume, da die zuständigen Schulbehörden das Programm vollständig mittragen; dagegen bestehen in manchen europäischen Ländern schon Bedenken, dass damit chinesischer Propaganda Zugang zu den Schulen gewährt werde. Um die Vielfalt der Konfuzius-Institute deutlich zu machen, brauchen eigentlich kaum noch die richtigen Sonderfälle ins Feld geführt zu werden: So ist das KonfuziusInstitut an der South Bank University in London spezialisiert auf Traditionelle Chinesische Medizin (TCM) und das Konfuzius-Institut in Singapur kann auf so umfangreiche Unterstützung der dort ansässigen chinesischen Gemeinde zurückgreifen, dass es praktisch auf Zuschüsse aus Beijing verzichten kann. Ein Spezialfall ist auch das KonfuziusInstitut in Hongkong - natürlich gibt es dort auch nach der Rückgabe an China eine ausländische Klientel, die Interesse an chinesischen Sprach- und Kulturprogrammen hat, aber da Hongkong auch als Sonderregierungszone heute fester Bestandteil der Volksrepublik China ist, mutet ein Konfuzius-Institut dort auch ein wenig an wie „Innere Mission".

Angesichts dieser Vielfalt und der schnellen Ausbreitung stehen die Konfuzius-Institute vor einer neuen Phase ihrer Entwicklung. Standen die Zeichen bislang vor allem auf Expansion und Quantität, werden in den kommenden Jahren Qualität und Verstetigung eine deutlich stärkere Rolle spielen: „Konfuzius“ soll sich zu einer wiedererkennbaren und unverwechselbaren Marke entwickeln.

\section{Das Konfuzius-Institut an der Heinrich-Heine-Universität Düsseldorf e. V.}

Düsseldorf hat seit vier Jahren ein Konfuzius-Institut. Es ist vieler Hinsicht eine Ausnahme. Während die meisten Institute in Deutschland an eine Sinologie angebunden sind, hat die Heinrich-Heine-Universität keine Chinastudien. Während üblicherweise auf deutscher Seite allein die jeweilige Universität als Partner auftritt, ist in Düsseldorf mit der Stadt ein weiterer Akteur mit großem Engagement beteiligt. Damit spielt das Konfuzius-Institut in Düsseldorf in mehrfacher Hinsicht eine wichtigere Rolle für Stadt und Umland als die Institute in Duisburg, Berlin oder Frankfurt. Es bietet einer breiten Öffentlichkeit einen Zugang zur chinesischen Sprache und Kultur und trägt dazu 
bei, Düsseldorf als einen der wichtigsten Chinastandorte in Deutschland zu etablieren. Gleichzeitig ist das Institut inzwischen auch wissenschaftlich tätig und hält Angebote für Studierenden der Heinrich-Heine-Universität bereit.

\section{Entstehung des Konfuzius-Instituts Düsseldorf}

Offiziell gegründet wurde das Konfuzius-Institut Düsseldorf im Dezember 2006. Seine Entstehung reicht aber weiter zurück - bis zum Oktober 2004: Der erste Anstoß, ein Konfuzius-Institut nach Düsseldorf zu holen, kam von Dr. Peter Hachenberg, Geschäftsführer des Sprachenzentrums und Leiter des Studiengebiets Deutsch als Fremdsprache an der Heinrich-Heine-Universität. Er hatte entsprechende Informationen aus China von einem alten Bekannten, Prof. Zhang Xiping, erhalten und wies den Leiter des Auslandsamtes der Universität, Dr. Werner Stüber, auf die damals gerade erst neu konzipierten Konfuzius-Institute hin. Zwar habe die Universität selbst in Sachen China (noch) nicht viel zu bieten, aber der Standort Düsseldorf sei „ziemlich ideal“ ${ }^{2}$ Da Oberbürgermeister Erwin die Beziehungen sehr ausdrücklich unterstütze, ergäben sich hier „wunderbare Perspektiven auch der Zusammenarbeit mit der Stadt Düsseldorf“. Stüber hielt dann am 21. Oktober 2004 in einem Vermerk die wichtigsten Informationen über das mögliche Vorgehen fest und formulierte noch vorsichtig, „ein Konfuzius-Institut an der Universität der Landeshauptstadt" könnte „als eine Facette zur Profilschärfung“ sinnvoll sein.

Der nächste Schritt folgte dann im Frühjahr 2005, dieses Mal bereits gemeinsam von deutscher und chinesischer Seite. Dr. Hachenberg hielt am 21. März einen Vortrag an der Pekinger Fremdsprachenuniversität (BFSU). Bei einer anschließenden Besprechung beschlossen Hachenberg und Zhang Xiaohui, damals Leiterin des Instituts für den Internationalen Austausch an der BFSU, sich gemeinsam für ein Konfuzius-Institut in Düsseldorf stark zu machen. Hachenberg sollte sich danach bemühen, die HeinrichHeine-Universität zu einem Antrag auf ein Institut zu bewegen. Gleichzeitig sicherte Zhang Unterstützung bei der Antragstellung zu und erklärte ihre Bereitschaft, einen Düsseldorfer Antrag beim National Office for Teaching Chinese as a Foreign Language $(\text { NOTCFL })^{3}$ schriftlich zu unterstützen und im direkten Kontakt zu vermitteln.

Hachenbergs Bemühungen in Düsseldorf stießen bei dem damaligen Rektor der HHUD, Prof. Dr. Dr. Alfons Labisch, auf offene Ohren. Labisch richtete am 4. Juli 2005 ein Schreiben an Xu Lin, damals noch Direktorin des NOTCFL und heute Generalsekretärin von Hanban, in dem er die Bedeutung Düsseldorfs als Landeshauptstadt und Wissenschaftsstandort hervorhob und Gespräche im Hinblick auf ein Konfuzius-Institut anbot. Fast gleichzeitig informierte der Rektor den Düsseldorfer Oberbürgermeister Joachim Erwin über seine Initiative und betonte, dass ein Konfuzius-Institut nicht nur das Profil der Universität schärfen, sondern auch zur Imagestärkung der Stadt Düsseldorf beitragen würde.

Schließlich stellte Labisch noch im Juli 2005 in einem weiteren Schreiben den Kontakt zur Chinesischen Botschaft in Berlin her und ersuchte den Botschafter Ma Canrong

\footnotetext{
2 Soweit nicht anders angegeben, stammen sämtliche Zitate aus den Akten des Konfuzius-Instituts Düsseldorf.

3 Hanban, das Hauptquartier der Konfuzius-Institute.
} 
um die Berücksichtigung Düsseldorfs bei der Standortwahl zukünftiger Konfuzius-Institute. Parallel leitete Hachenberg die Gespräche auf der operativen Ebene ein. Er setzte die Botschaftsrätin Dr. Liu Jinghui vom Stand der Dinge in Sachen Konfuzius-Institut für Düsseldorf in Kenntnis. Bei seiner Schilderung von Düsseldorf als idealem Standort für ein chinesisches Kulturinstitut konnte er bereits auf die „China-goes-DUS“-Initiative der Stadt verweisen. Hintergrund für die schnelle Vorsprache waren nicht zuletzt Nachrichten, dass Düsseldorf bereits Konkurrenz hatte - Stüber schrieb in einem Vermerk, dass sich die Ruhr-Universität Bochum gleichfalls um ein Konfuzius-Institut bemühe und Düsseldorf entsprechend nachdrücklich in Position gebracht werden müsse.

Der nächste wichtige Schritt erfolgte in Beijing im September 2005. Es war wieder Hachenberg, der sich gemeinsam mit Zhang Xiaohui, Zhang Xiping und Li Xuetao von der BFSU zu einem Gespräch mit Wang Luxin traf, dem Direktor der Abteilung für internationalen Austausch beim NOTCFL. Anknüpfend an das Schreiben des Düsseldorfer Universitätsrektors formulierte Hachenberg das Interesse Düsseldorfs an einem Konfuzius-Institut. Er hob dabei sowohl die akademische Qualität der HHUD hervor, wie auch den Stellenwert des Wirtschaftsstandorts Düsseldorf gerade im Hinblick auf die deutsch-chinesischen Wirtschaftsbeziehungen. Verweisen konnte er zu diesem Zeitpunkt bereits darauf, dass Universität und Stadt an einem Strang zogen - OB Erwin hatte inzwischen auch den Willen der Stadt zum Ausdruck gebracht, ein KonfuziusInstitut für Düsseldorf zu unterstützen. Wang verwies zunächst auf die überraschende Resonanz, die diese chinesische Initiative aus aller Welt erfahren hatte. Man komme mit den Gründungen kaum nach und müsse erst einmal die bereits errichteten Institute konsolidieren. Anschließend machte er deutlich: „Er könne aber von Seiten des NOTCFL die Gründung eines Konfuzius-Instituts in Düsseldorf im Zeitraum von etwa einem Jahr zusagen. " Mit Düsseldorf solle ein neuer Typ von Institut ins Leben gerufen werden: Nicht gebunden an eine akademische Sinologie und ein sinologisches Institut wie in Berlin, sondern mit einem Fokus auf Sprachkursen gerade für Kunden aus der Wirtschaft, und dafür sei Düsseldorf der ideale Standort.

Wang kam ebenfalls auf die Situation in Deutschland und die mögliche Konkurrenz für Düsseldorf zu sprechen. Auch er sah Bochum als ernsthaften Rivalen, da die Universität zum einen über eine nennenswerte Sinologie verfüge und zudem das Landesspracheninstitut (LSI) mit seinem Sinicum beherberge. Allerdings meinte Wang gleichzeitig, dass genau diese beiden Punkte auch entscheidende Nachteile für Bochum sein könnten. Da es zu diesem Zeitpunkt gerade darum ging, ein Konfuzius-Institut neuen Typs aufzubauen, unabhängig von einer akademischen Sinologie, in einem wirtschaftlich interessanten Umfeld, das gerade Düsseldorf biete.

Hachenberg empfahl nach seiner Rückkehr aus Beijing, schnell zu handeln. Der Moment schien günstig, nun galt es, die Gelegenheit zu nutzen - nach seiner Einschätzung bestanden jetzt realistische Aussichten auf ein Düsseldorfer Konfuzius-Institut. Man sollte so schnell wie möglich an die Botschaft, und hier an die damalige Leiterin der Erziehungsabteilung der Botschaft in Berlin, Liu Jinghui, herantreten. Sie sollte nach Düsseldorf eingeladen werden, um sich ein Bild von Stadt und Universität machen zu können. Dabei sollten beide, Stadt und Universität, eng zusammenarbeiten. Stüber übernahm diese Empfehlung und regte an, Liu Jinghui direkt in Berlin aufzusuchen. Mit der bald durchzuführenden Reise sollte wieder Hachenberg beauftragt werden, der auf- 
grund seiner hervorragenden Chinakenntnisse dafür bestens geeignet sei. Schließlich reisten beide nach Berlin.

Dieser Besuch fand dann im Januar 2006 statt. Liu Jinghui sah in Düsseldorf als herausragendem Dienstleistungs- und Industriestandort in dem bevölkerungsreichsten deutschen Bundesland einen denkbar günstigen Ort für ein Konfuzius-Institut. Dies bezog sich auf die regionale Verteilung der Institute in Deutschland: Weitere Bewerbungen lagen zwar aus Hamburg, Heidelberg und Frankfurt am Main vor, die einzige regionale Konkurrenz für Düsseldorf sei aber Bochum. Liu sagte zu, in Kürze Gespräche mit den zuständigen Instanzen in Beijing zu führen und forderte die HHUD auf, nun auch einen formalen Antrag an die Botschaft zu stellen. Der positive Gesamteindruck wurde noch unterstrichen, als im gleichen Monat ein Schreiben aus Beijing eintraf, in dem Xu Lin als Direktorin des NOTCFL den Wunsch der HHUD, in Düsseldorf ein KonfuziusInstitut einzurichten, ausdrücklich begrüßte. Auf Anregung von Stüber und Hachenberg suchte die HHUD nun den engen Schulterschluss mit der Stadt. Universitätsrektor Labisch wandte sich Anfang Februar 2006 erneut an OB Erwin und schilderte ihm die Situation. Im Besonderen sollten Stadt und Universität geeignete Räumlichkeiten möglichst kostenfrei zur Verfügung stellen und der OB parallel zur Universität Kontakt zur Botschaft aufnehmen.

Fast zur gleichen Zeit teilte die chinesische Botschaft am 8. März aber mit, dass ein weiterer Konkurrent hinzugekommen war: Köln hatte ebenfalls ein Konfuzius-Institut beantragt. Stüber empfahl eine möglichst rasche Reaktion. Ein Angebot der Stadt Düsseldorf und ein detaillierter Antrag sollten nicht mehr abgewartet werden, sondern sofort ein formaler Antrag auf den Weg gebracht werden. So geschah es, bereits am folgenden Tag beantragte Universitätsrektor Labisch per Schreiben an Botschaftsrätin Liu die Einrichtung eines Konfuzius-Instituts in Zusammenarbeit mit der HHUD und kündigte die baldige Übersendung des vollständigen Düsseldorfer Angebots an. Zur Zusammenarbeit mit der Stadt konnte immerhin darauf verwiesen werden, dass erste Schritte zur Sichtung geeigneter Räumlichkeiten unternommen worden waren.

Die Stadt zog in der Tat mit. Am 14. März schrieb OB Erwin an Rektor Labisch und begrüßte die Ergänzung der wirtschaftlichen Zusammenarbeit der Stadt Düsseldorf mit China um eine kulturelle Ebene und bestärkte die Universität, das Konfuzius-Institut zu beantragen. Erwin sagte die Unterstützung der Stadt zu und bat um ein entsprechendes Kooperationskonzept. Zwei Wochen später unterstrich Erwin das städtische Engagement mit einem Memorandum unter dem Titel „China-Standort Düsseldorf: Mit einem Konfuzius-Institut gemeinsam zu größerem Erfolg!“

Darin wurde das Düsseldorfer China-Engagement und die bereits vorhandenen Institutionen und Initiativen zusammengefasst. Die Ausführungen gipfelten in dem Schluss, das Konfuzius-Institut sei eine „ideale Ergänzung der vorhandenen Strukturen“. Mit dem Wirtschaftsstandort Düsseldorf wachse auch die Nachfrage nach Informationen über China, seine Geschichte und Kultur. Hinzu käme ein stark wachsendes Interesse an der chinesischen Sprache. Das Institut wäre somit eine hervorragende Ergänzung und würde eine Lücke in dem Chinakonzept der Stadt schließen. In Zusammenarbeit mit der HHUD ließe sich das Konzept des Konfuzius-Instituts harmonisch mit dem wirtschaftlichen und kulturellen Leben der Region verbinden. Erwin fasste zusammen: 
Unsere Arbeit basiert auf der grundsätzlichen Idee, dass wirtschaftliche Kooperation nur auf der Basis von gegenseitigem kulturellem Verständnis gedeihen kann. Deshalb unterstützen wir chinesische und deutsche Unternehmen dabei, gegenseitig mehr über einander zu lernen, sich nicht nur mit harten Wirtschaftsfakten, sondern auch mit kulturellen, historischen und gesellschaftlichen Fragen zu beschäftigen. Die Unterstützung von chinesischen Einrichtungen, die chinesische Sprache und Kultur in Düsseldorf vorstellen und heimisch machen sollen, ist für die Stadt eine wichtige Aufgabe.

Im Hintergrund arbeiteten beide Seiten nun intensiv an einem tragfähigen Konzept zur Einrichtung des Instituts. Die Stadt übernahm die Miete des Instituts für einen Zeitraum von zunächst drei Jahren. Im Gegenzug sollte die Universität für die Nebenkosten aufkommen sowie das notwendige Mobiliar finanzieren. Bei der Suche nach geeigneten Räumlichkeiten arbeiteten Stadt und Universität eng zusammen. Im April konnte Rektor Labisch die chinesische Botschaft erfreut über die großen Fortschritte auf Düsseldorfer Seite informieren: Sowohl die Rah-

RHEINISCHE POST SAMSTAG 3. JUNI 2006

\section{Konfuzius baut Brücken am Rhein}

\begin{abstract}
(tler) Was dem Deutschen sein Goe- Labisch, Rektor der Heine-Uni, der the-Institut, steht in China unter num eine Absichtserklärung zur Inder Schirmherrschaft von Konfuzi- stitutsgründung unterzeichnete. der Schirmherrschaft von Konfuzi- stitutsgrundung unterzeichnete.
us: eine Einrichtung, die Sprache Weitere internationale Unternehund Kultur desHeimatlandes indie men dürften so auf die LandesWelt hinausträgt und für interkul- hauptstadt aufmerksam werden. turelle Kommunikation wirbt. Kooperieren werden in erster LiRund 70 Konfuzius-Institute gibt es nie das Sprachenzentrum der Uni weltweit. Nach Berlin und Erlan- und đie Beijing Foreign Studié gen-Nurnberg soll Disseldorf nun und die gen-Nurnberg soll Düsseldorf nun University. „Sprache ist eine Brücke der erste-und einzige-Standort in für globales Denken", so Jinghu NRW werden. Liu, Bildungsexpertin von der chiVon der sprachlichen und kultu- nesischen Botschaft. Mit im Boot ist rellen Kompetenz des Instituts wird die Stadt, die die Răume zur Verfüauch der Wirtschaftsstandort Düs- gung stellen wird. In diesem Jahr seldorf profitieren", sagte Alfons soll das Institut erơffnen.
\end{abstract}

Abb. 1: Meldung der „Rheinischen Post“ von 3. Juni 2006 menvereinbarung zwischen Hanban, HHUD und Stadt Düsseldorf, wie auch eine Vereinbarung mit der BFSU standen kurz vor dem Abschluss.

Am 27. Juni 2006 konnte schließlich die offizielle Rahmenvereinbarung über die Einrichtung eines Konfuzius-Instituts in Düsseldorf unterzeichnet werden. Die offiziellen Partner waren hier Botschaft und BFSU von chinesischer Seite, auf der deutschen Seite Stadt Düsseldorf und HHUD. Die Universität wurde laut Rahmenvereinbarung im Besonderen durch das Universitätssprachenzentrum vertreten, an dem bis heute die universitären Chinesischkurse angesiedelt sind. Im Sommer 2006 titelte die Rheinische Post schon „Konfuzius-Institut kommt nach Düsseldorf“ ${ }^{4}$ Die Zeitung brachte das breite Chinainteresse auf den Punkt: „Jeder spricht über China, über dessen Wirtschaftszuwachs im fast zweistelligen Bereich, dessen hocheffiziente Arbeitskräfte und über dessen noch unerschöpftes Potential. Nur Chinesisch kann kaum einer sprechen." China starte nun auch eine Kulturoffensive, um mit den Konfuzius-Instituten Chinesisch zu verbreiten. Nun wolle auch die Heinrich-Heine-Universität ein Institut einrichten, damit werde Düsseldorf der dritte Konfuzius-Standort in Deutschland. Das Fazit lautete: „Das Institut soll im nächsten Semester in Düsseldorf gegründet werden. Der Erfolg ist ihm sicher. China liegt im Trend.“

War die Einrichtung des Konfuzius-Instituts damit unter Dach und Fach, konnten die aufwendigen praktischen Vorbereitungen nun erst richtig beginnen. Die Räumlichkeiten in der Graf-Adolf-Straße waren bereits gefunden, sie mussten aber erst komplett neu eingerichtet werden. Zur gleichen Zeit war auch die Frage der zukünftigen Leitung des neuen Instituts zu klären. Erster chinesischer Direktor wurde Li Xuetao, erster deut-

\footnotetext{
4 Vgl. Schnettler (2006).
} 


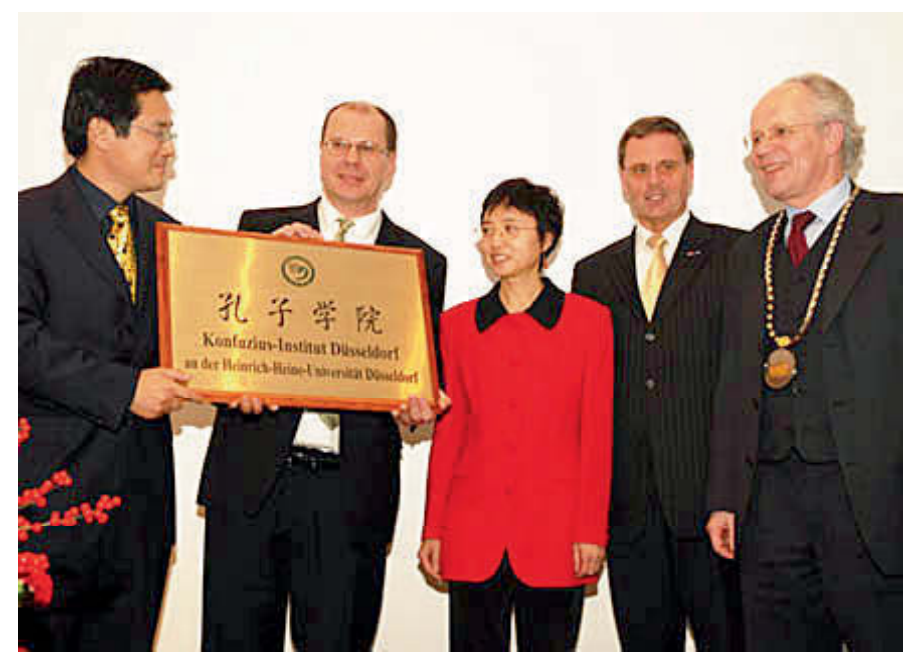

Abb. 2: Übergabe der offiziellen Messingtafel bei der Eröffnung des Konfuzius-Instituts Düsseldorf. Von links nach rechts: Li Xuetao, Peter Hachenberg, Liu Jinghui, Wilfried Kruse, Alfons Labisch

scher Direktor Dr. Hachenberg - beide waren von Beginn an in das Zustandekommen des Konfuzius-Institut Düsseldorf involviert gewesen und damit eine hervorragende Wahl, um der neuen Institution einen guten Start zu garantieren. Hachenberg blieb Geschäftsführer des Sprachenzentrums und Leiter des Bereichs Deutsch als Fremdsprache. Er wurde zwar von einigen Unterrichtsverpflichtungen befreit, aber es zeugt von seinem erheblichen Engagement für die Sache des Konfuzius-Instituts, dass er bereit war, zusätzlich noch die Position des deutschen Direktors zu übernehmen.

Der offizielle Gründungsakt des Konfuzius-Instituts Düsseldorf (KID) fand am 6. Dezember 2006 statt und nahm fast den ganzen Tag in Anspruch. Erster Programmpunkt war die formale Gründung des Vereins „Konfuzius-Institut Düsseldorf an der Heinrich-Heine-Universität e. V.“, bis heute der Träger des Instituts. Passend zu dem deutschchinesischen Charakter der neuen Einrichtung wurde auch der Vorstand des Vereins paritätisch besetzt. Die Unterzeichnung der Vereinssatzung wurde in der HHUD vorgenommen, die erste Vorsitzende wurde die damalige Prorektorin für Internationales, Professorin Dr. Vittoria Borsò.

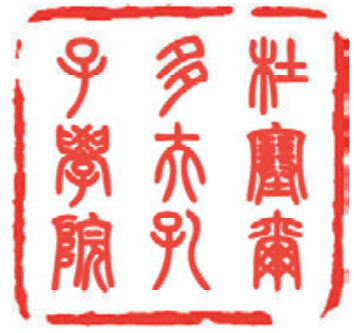

Abb. 3: Siegel des Düsseldorfer Konfuzius-Instituts

Im Anschluss ging es zur Einweihungsfeier ins Düsseldorfer Zentrum. Die neuen Büroräume in der Graf-Adolf-Straße 63 erwiesen sich für den Festakt bald als zu klein, einen Monat vorher lagen schon über 100 Anmeldungen vor. Dank der Firma Ernst \& Young konnte die Veranstaltung ohne große Schwierigkeiten ins GAP 15 verlegt werden. Zur Eröffnung sprachen Universitätsrektor Prof. Labisch, der Beigeordnete Wilfried Kruse als Vertreter des Oberbürgermeisters Erwin und als Vertreterin Chinas die damalige Leiterin der Erziehungsabteilung der Botschaft in Berlin, Liu Jinghui. Im An- 
schluss gab es ein Festbankett mit Unterhaltungsprogramm im Düsseldorfer China-Center.

Zur Eröffnung übersandte Xu Lin als Leiterin des Office of Chinese Language Council International ihre Glückwünsche und zitierte aus einem Gedicht von Heinrich Heine:

Da mochtest du das arme Reis beklagen,

An deinem gütgen Worte läßt du es ranken,

Und dir, mein hoher Meister, sollt ich's danken,

Wird einst das schwache Reislein Blüten tragen.

Das schwache Reislein werde sicher unter den gemeinsamen deutschen und chinesischen Bemühungen zu einem großen Baum heranwachsen und „reiche Früchte der Freundschaft zwischen beiden Ländern tragen“. Prof. Labisch blickte als Rektor der HHUD in seiner Rede zur Eröffnung auf die Entstehungsgeschichte des Instituts zurück und sah in Heinrich Heine einen Düsseldorfer Faktor, der sicher auch zur Wahl des Standorts beigetragen hatte:

Die Heinrich-Heine-Universität ist ihrem Namenspatron verpflichtet: brillanter Intellekt, weltoffener Geist und weites Herz, Toleranz gegenüber anderen Menschen und Meinungen, Engagement für den Kulturraum Europa und den weltweiten kulturellen Austausch und für die Stadt Düsseldorf. ${ }^{5}$

Es sei der HHUD damit ein besonderes Anliegen, international grenzüberschreitend präsent zu sein und den Herausforderungen einer globalen Wissens- und Informationsgesellschaft zu begegnen, indem gemeinsam mit internationalen Partnern Konzepte und Praktiken einer umfassenden Bildung umgesetzt werden, bei denen der kulturvermittelnde Blick auch in andere Hemisphären richtungsweisend ist. Für den Aufbau der neuen Einrichtung hätten sich nun ideale Partner gefunden: Gerade weil mit dem Düsseldorfer Konfuzius-Institut neue Wege beschritten werden sollten, seien mit dem Zusammenspiel von Universität und Stadt Düsseldorf „mit ihrer wirtschaftlichen Kraft und kulturellen Attraktivität“ optimale Voraussetzungen für den Aufbau eines wichtigen Kulturvermittlers gegeben. Abschließend dankte Labisch mit Hachenberg und Stüber den beiden Akteuren, ohne die das Institut nicht zustande gekommen wäre.

\section{Die Entwicklung des Düsseldorfer Konfuzius-Instituts seit seiner Gründung}

Eine erste Bilanz zog das Konfuzius-Institut etwas mehr als ein Jahr nach seiner Gründung im Januar 2008. Zu diesem Zeitpunkt konnte die junge Kulturinstitution bereits auf einige Erfolge zurückblicken und erfreut feststellen, dass sich das KID „bereits fest im kulturellen Leben der Stadt und im akademischen Leben der Universität verankert" hat und vielfältige Kontakte schon zur lokalen Wirtschaft geknüpft waren. Kernkomponente des Programms waren und sind bis heute die Sprachkurse, an denen 2007 fast 300 Personen teilgenommen hatten. Hervorzuheben sind auch die Chinesischkurse für Schülerinnen und Schüler des Düsseldorfer Görres-Gymnasiums, die über mehrere Semester im Konfuzius-Institut stattfanden. Hinzu kam im Kulturprogramm eine Reihe von Vorträgen namhafter deutscher Sinologen wie des Bonner Professors Wolfgang Kubin über Konfuzius und des Direktors der Herzog-August-Bibliothek in Wolfenbüttel,

5 Rede zur Eröffnung des Konfuzius-Instituts (Archiv KID). 
Helwig Schmidt-Glintzer, über den Pluralismus in den chinesischen Religionen. Darüber hinaus wurden Veranstaltungen zu wirtschaftlichen und politischen Themen in Zusammenarbeit mit der Industrie- und Handelskammer zu Düsseldorf und dem China-Kompetenzzentrum des Wirtschaftsförderungsamtes durchgeführt. Besonders hervorzuheben ist die Lesung von Bei Dao, des derzeit wohl berühmtesten chinesischen Lyrikers, im Juli 2007. Weitere Höhepunkte des Programms waren ein Konzert zum 100-jährigen Geburtstag des Violinisten und Geigenbauers Tan Shuzhen und die Beteiligung an der Düsseldorfer Märchenwoche mit einer Lesung chinesischer Märchen die seitdem zu einer guten Tradition geworden ist. Genauso kontinuierlich hat sich das Konfuzius-Institut seit 2007 an der Durchführung des Kreativwettbewerbs Chinesisch beteiligt. Dieser Wettbewerb etablierte sich als größte Veranstaltung zur chinesischen Sprache im gesamten Bundesgebiet. Dabei ist bis heute vor allem die vertrauensvolle Zusammenarbeit mit dem Ministerium für Schule und Weiterbildung hervorzuheben. Mit der verantwortlichen Ministerialrätin Henny Rönneper arbeitet das KID bis heute eng zusammen.

Einen wichtigen Schritt machte das KID im September 2007. Auf der Vorstandssitzung am 20. September wurde die Vereinssatzung präzisiert. Der Vereinszweck besteht danach in der Durchführung von Chinesisch-Kursen, von wissenschaftlichen und kulturellen Veranstaltungen und der Förderung der Begegnung von Deutschen und Chinesen sowie in der Betreuung chinesischer Besucher in Deutschland. Diese Satzungsänderung war nicht nur die Formulierung des bereits vorhandenen Arbeitsprogramms, sondern schuf auch die Voraussetzungen für die offizielle Anerkennung der Gemeinnützigkeit des Konfuzius-Instituts.

Im November 2007 beantragte das Konfuzius-Institut, seiner Verbindung mit der HHUD nun auch einen offiziellen Status zu verleihen, wie es auch im Rahmenvertrag vereinbart worden war. In der Rektoratssitzung am 6. März 2008 wurde das KID offiziell als An-Institut anerkannt. Der erste Wechsel in der Institutsleitung erfolgte im Januar 2008. Li Xuetao legte sein Amt als chinesischer Direktor nieder, da er in Beijing in der BSSU neue Aufgaben übernahm. Seine Nachfolgerin wurde Deng Xiaojing, die am 31. Januar 2008 zur neuen chinesischen Direktorin gewählt wurde. Sie trat ihren Dienst dann im April des Jahres an.

Im Jahr 2008 setzte das KID die erfolgreiche Arbeit fort. Zum Ausbau des differenzierten Sprachkursprogramms und des umfangreichen Programms von Vorträgen, Lesungen, Ausstellungen und Konzerten kamen unter anderem Kooperationen mit dem Düsseldorfer China-Center (DCC) und dem Kulturamt der Stadt Düsseldorf. Besonders die Zusammenarbeit mit der Stadt entwickelte sich erfolgreich weiter. Das Kulturamt unterstützte ein Projekt zu „Heine und China“ sowie einen „Kulturworkshop China“. Der Sprachbereich bildet bis heute den Kern der Arbeit am KID. Das gilt nicht nur für die Kurse am Institut, sondern gleichermaßen auch für das Programm an der Universität, das seit Gründung des KID in enger Zusammenarbeit mit dem Sprachenzentrum angeboten und umgesetzt wird. Die universitären Sprachkurse erfreuen sich eines wachsenden Interesses. Im Sommersemester 2010 besuchten fast 90 Studierende die fünf angebotenen Kurse, im Wintersemester 2010/2011 sind es bei sechs Kursen über 110 Teilnehmerinnen und Teilnehmer. 


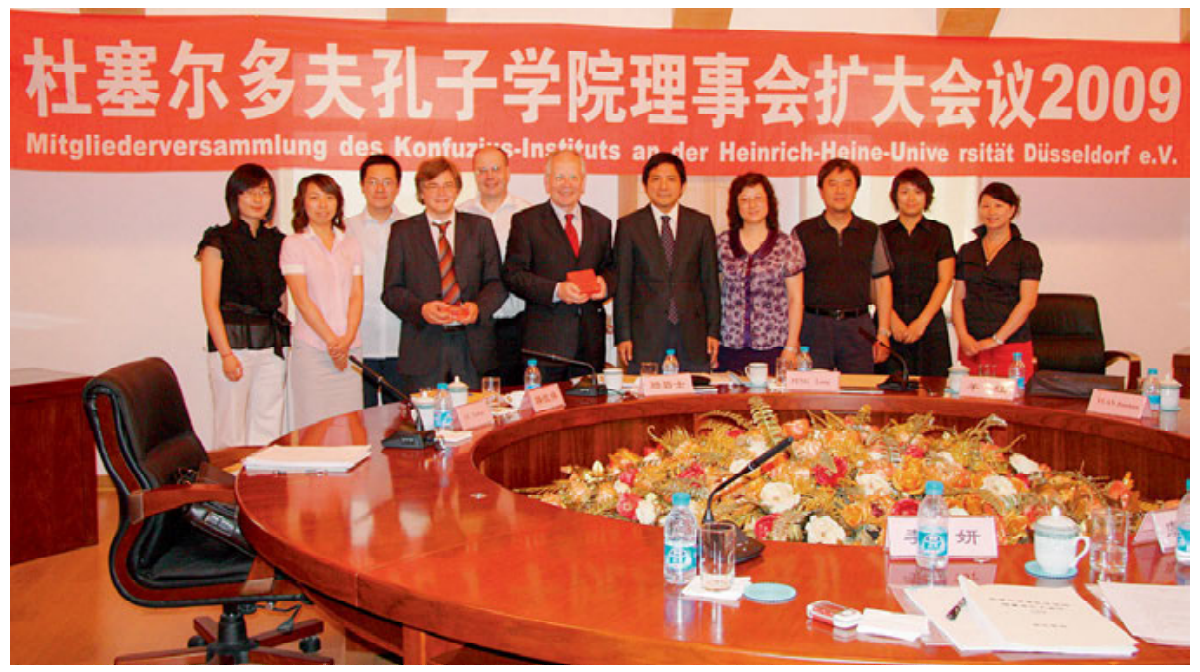

Abb. 4: Mitgliederversammlung in Peking. Von links nach rechts: Yu Xiangrong, Gong Jing, Li Xuetao, Elmar Schafroth, Peter Hachenberg, Alfons Labisch, Peng Long, Zhang Xiaohui, Yuan Jianhua, Li Yan, Deng Xiaojing

Im Juli 2009 fand die erste Mitgliederversammlung bei dem chinesischen Partner BFSU statt. In Beijing wurden wichtige Änderungen für das Institut beschlossen. Der alte Vorstand trat zurück. Allerdings blieben alle dem Verein als Mitglieder erhalten. Es wurden vier neue Mitglieder aufgenommen: Prof. Peng Long, Vizepräsident der BFSU, Professorin Zhang Xiaohui, Dekanin der Abteilung Konfuzius-Institute der BFSU, Li Yan als Nachfolgerin von Deng Xiaojing als chinesischer Direktorin, und schließlich Prof. Dr. Peter Proksch von der HHUD. Neuer Vorstandsvorsitzender wurde Prof. Labisch, Förderer des KID seit Anbeginn. Die Neumitglieder Peng und Proksch wurden zu stellvertretenden Vorsitzenden gewählt, weitere Vorstandsmitglieder wurden Li Yan, Li Xuetao und Professorin Borsò, die schon dem ersten Vorstand angehört hatte.

Ein weiterer wichtiger Wechsel bahnte sich auf dieser Mitgliederversammlung an: Dr. Hachenberg bereitete seinen Rückzug vom Amt des deutschen Direktors vor. Hatte er bereits auf der Mitgliederversammlung im November 2008 erklärt, nach Ablauf seiner Amtsperiode nicht mehr zur Verfügung zu stehen, erklärte er in Beijing nun auch formal seinen Rücktritt. Da aber zu diesem Zeitpunkt noch kein Nachfolger für seine Position benannt werden konnte, erklärte sich Hachenberg bereit, den Posten kommissarisch weiter wahrzunehmen - allerdings längstens bis zum 31. Dezember 2009.

Zur Sicherstellung der Kontinuität am KID und dessen Fortentwicklung waren insbesondere zwei Probleme zu regeln: Die Verlängerung der Rahmenvereinbarung zwischen Hanban, Heinrich-Heine-Universität sowie der Stadt Düsseldorf und die Einrichtung einer Stelle für den deutschen Direktor des Instituts.

Im Februar 2009 wurde dem neu gewählten Rektor Prof. Dr. Dr. H. Michael Piper ein Antrag auf Verlängerung der Rahmenvereinbarung vorgelegt. Die Antragsteller konn- 
ten auf eine erfolgreiche Arbeit des Instituts und ein mustergültiges Projekt hinweisen. Mit dem Konfuzius-Institut stelle sich die Universität mitten in der Stadt „als Repräsentantin von Internationalität und der Zusammenarbeit mit China dar." Inzwischen wurden bereits Verhandlungen mit Hanban über die Finanzierung einer vollen Stelle für den deutschen Direktor geführt. Gerade die erfolgreiche Etablierung des Instituts mit dem stetig wachsenden Umfang und der zunehmenden Komplexität der Aufgaben machte die Bereitstellung einer angemessen ausgestatteten Position erforderlich. Während die Rahmenvereinbarung von Stadt und Universität seit dem Frühjahr 2009 ohne großes Aufheben fortgeführt wurde, zog sich die Bewilligung der Stelle des deutschen Direktors deutlich länger hin. Im Januar 2010 hat das Konfuzius-Institut Düsseldorf mit Dr. Cord Eberspächer als erstes in Deutschland einen hauptamtlichen Direktor bekommen. Damit ergeben sich deutlich erweiterte Möglichkeiten für mehr Angebote zu chinesischer Sprache und Kultur, Aktivitäten um wissenschaftlichen Bereich, aber auch Angeboten zur Chinaberatung und zum interkulturellen Training.

\section{Der große Sprung nach vorn}

Die neue Leitung des KID mit dem Vorstandsvorsitzenden Prof. Dr. Dr. Labisch, der chinesischen Direktorin Li Yan und dem deutschen Direktor Dr. Eberspächer hat im Frühjahr 2010 ein strategisches Konzept für die Entwicklung des Instituts vorgelegt. Als „Großer Sprung nach vorn“ umreißt dieses Programm die Planungen für die kommenden fünf Jahre. Danach besteht das Fundament der Arbeit aus vier Säulen, die kurz mit den Begriffen Sprache, Kultur, Wissenschaft sowie Wirtschaft und Politik charakterisiert werden können.

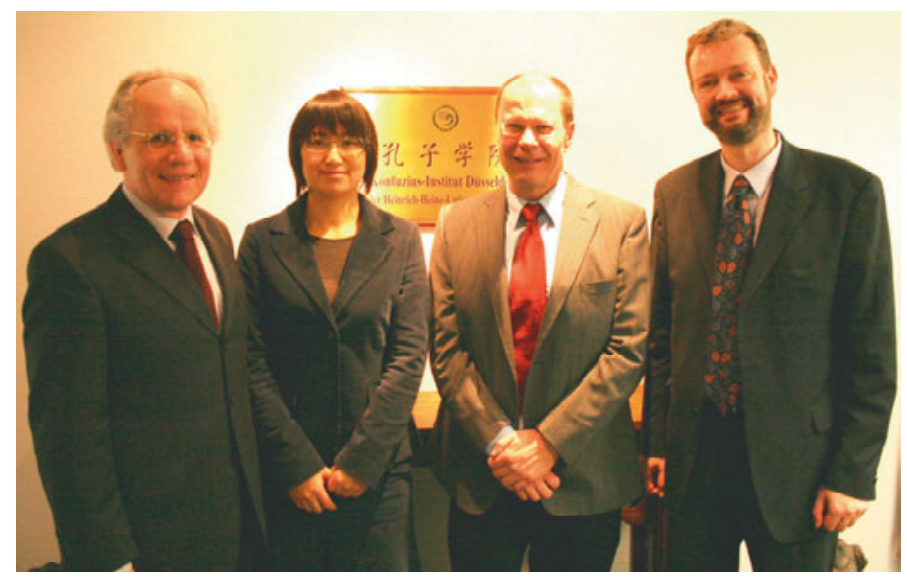

Abb. 5: Konfuzius-Institut unter neuer Leitung. Von links nach rechts: Alfons Labisch, Li Yan, Peter Hachenberg, Cord Eberspächer

Die ersten beiden Säulen beinhalten vor allem die Fortführung der erfolgreichen Arbeit der ersten drei Jahre. Die bestehenden Programme sollen lediglich mit neuen Angeboten oder weiteren Kooperationen vermehrt und verbreitert werden. Der Sprach- 
bereich ist ausbaufähig, hier kann zum einen das Angebot wie auch die Kooperation mit den Schulen in Düsseldorf und im Umland erweitert werden. Zum anderen wurden die Kurse für Geschäftschinesisch noch nicht in dem Umfang bekannt gemacht und genutzt, wie es der starken Chinaorientierung des Standorts Düsseldorf entsprechen sollte. Das Kursprogramm an der HHUD soll ebenfalls in Zusammenarbeit mit dem Sprachenzentrum weiter entwickelt werden, auch im Hinblick auf einen eventuellen Austausch von Studierenden mit chinesischen Partneruniversitäten. Die offizielle chinesische Sprachprüfung Hanyu Shuiping Kaoshi (HSK) findet ein deutlich gesteigertes Interesse. Hier ist das KID Anlaufstelle für Prüfungskandidaten aus ganz NordrheinWestfalen. Diese Position gilt es auszubauen. Dabei wird das KID verstärkt mit dem Schulministerium und der Düsseldorfer Bezirksregierung zusammenarbeiten. Die Einrichtung von Konfuzius-Klassenräumen ist eine weitere Aufgabe für die kommenden Jahre. Das KID strebt zunächst die Verwirklichung eines Modellprojekts mit einer Düsseldorfer Schule an.

Im Kulturbereich bietet das KID auch weiterhin eine bewährte Mischung aus Vorträgen, Lesungen, Ausstellung und ähnlichen Veranstaltungen. Das KID wird in der Zukunft mehr Wert auf Veranstaltungsreihen legen. So ist an die Einrichtung eines Jour fixe gedacht, bei dem einmal im Monat verschiedene Chinathemen nach einem Impulsvortrag diskutiert werden. Die Kulturveranstaltungen sind kooperative Veranstaltungen. Die Palette der Institutionen, mit denen das KID bisher zusammenarbeitet, wie beispielsweise das Literaturbüro NRW oder die Volkshochschule, ist in diesem Jahr bereits um das Hetjens-Museum erweitert worden. Der Dialog mit möglichen Partnern soll verstetigt und intensiviert werden. So gibt es seit dem Frühjahr 2010 ein Treffen der „Chinavereine“, bei dem sich Vertreter von KID, dem Düsseldorfer China-Center, der Gesellschaft für Deutsch-Chinesische Freundschaft Düsseldorf, der Deutsch-Chinesischen Gesellschaft Neuss und der Asian Social Business Community austauschen. Aus diesen Treffen sind bereits mehrere Veranstaltungsideen hervorgegangen.

Die dritte Säule unter dem Stichwort Wissenschaft ist weitgehend neu konzipiert. Dieser Bereich bündelt eine ganze Reihe von Aktivitäten, in denen sich das KID stärker engagieren und profilieren will. Dazu gehört zunächst eine engere Zusammenarbeit mit der Heinrich-Heine-Universität. Da die Düsseldorfer Universität keine Chinastudien hat, steht das KID hier in einer besonderen Verantwortung. Die bislang bereits angebotenen Sprachkurse werden sukzessiv durch ein Seminarangebot ergänzt, das Studierenden im Rahmen des Studium Universale Möglichkeiten bietet, sich noch intensiver mit China auseinanderzusetzen. Nach einem Kalligraphiekurs im Sommersemester 2010, der als erster Versuch sehr gut angenommen wurde, wird im Wintersemester 2010/2011 eine Vorlesungsreihe zur chinesischen Philosophie angeboten. Das KID sieht sich gleichzeitig als Ansprechpartner und Vermittler für Ausbau und Intensivierung der Chinakontakte der Universität insgesamt. Das gilt für den Studierendenaustausch genauso wie für die Suche nach Partnern für gemeinsame, internationale Forschungsprojekte. Im Rahmen seiner Möglichkeiten beteiligt sich das KID auch direkt an wissenschaftlichen Forschungsvorhaben. So besteht bereits eine Kooperation mit dem Institut für die Geschichte der Medizin bei der Anbahnung des Forschungsrahmens „Multiple Modernitäten“, wobei das KID bereits geholfen hat, Kontakte zur Shanghaier Jiaotong-Universität zu knüpfen. Den eigenen wissenschaftlichen Aktivitäten will das KID in Zukunft auch 
eine eigene Plattform schaffen. Erträge der Forschung zur chinesischen Kultur und Geschichte sollen in einer Publikationsreihe einer breiten Öffentlichkeit nahe gebracht werden.

Die vierte Säule deckt unter den Stichworten Politik und Wirtschaft eigentlich zwei Bereiche ab. Dabei dreht es sich ausdrücklich weder um politische noch wirtschaftliche Aktivitäten des KID. Das Institut arbeitet aber an Angeboten, die seiner Verantwortung für Düsseldorf als starkem chinesischem Wirtschaftsstandort gerecht werden. Neben der Ausweitung und besseren Bekanntmachung der bereits vorhandenen Angebote für Wirtschaftschinesisch wird das KID ein Programm für interkulturelle Beratung und Training aufbauen. Dabei wird sich das Institut auf seine Kernkompetenzen in Sprache und Kultur konzentrieren und möchte damit vorhandene Angebote im rein wirtschaftlichen Bereich systematisch ergänzen. Es geht dem KID in seiner Arbeit um eine gezielte Vernetzung. Das betrifft zunächst die Konfuzius-Institute selbst. Angesichts der kurzen Geschichte der Konfuzius-Institute verwundert es kaum, dass sich bislang unterhalb der Ebene des Hauptquartiers Hanban in Beijing noch keine nennenswerten Strukturen herausgebildet haben. Inzwischen entwickelt sich aber ein Dialog zwischen den in der Bundesrepublik und Europa aufgebauten Instituten, der in der nahen Zukunft auch zu einer stärkeren Zusammenarbeit führen wird. Diese Zusammenarbeit wird vom KID mitgetragen und mitgestaltet. Die Konfuzius-Institute sollen zu einem Markenzeichen entwickelt werden, das für qualitativ hochwertige Angebote und verlässlichen Service für alle steht, die mit China steht zusammenarbeiten.

Diese Qualifikationen hat sich auch das KID auf seine Fahnen geschrieben. Es wird durch seine Arbeit seinen Bekanntheitsgrad in Düsseldorf und in ganz NRW weiter ausbauen. Mit seinem Standort in der Hauptstadt des größten Bundeslandes ist es Ansprechpartner auf Landesebene - auch im Bereich der offiziellen Chinesischprüfung HSK.

\section{Literatur}

Hachenberg, Peter und Xuetao Li (2007). „Das Konfuzius-Institut Düsseldorf an der Heinrich-Heine-Universität Düsseldorf e. V. - Gründung, Programm und Perspektiven“, in: Alfons LABISCH (Hrsg.). Jahrbuch der Heinrich-Heine-Universität Düsseldorf 2006/2007. Düsseldorf, 533-541.

SChnettler, Jan (2006). „Konfuzius-Institut kommt nach Düsseldorf“, Rheinische Post vom 4. August 2006. 
